\title{
MODELAGEM MATEMÁTICA DE SECAGEM DE BANANA EM DIFERENTES ESTÁDIOS DE MATURAÇÃO
}

\author{
L. B. de CARVALHO ${ }^{* 1}$, J. R. J. JUNQUEIRA ${ }^{1}$, A. M. P. BRAGA ${ }^{2}$, M. HOCHHEI ${ }^{2}$, M. A. \\ SILVA $^{2}$ e J. L. G. CORRÊA ${ }^{1}$ \\ ${ }^{1}$ Universidade Federal de Lavras, Departamento de Ciência dos Alimentos \\ ${ }^{2}$ Universidade Estadual de Campinas, Faculdade de Engenharia Química \\ *E-mail para contato: lucasbcarv@gmail.com
}

\begin{abstract}
RESUMO - A banana (Musa ssp.) apresenta elevado potencial de produção e consumo no mundo, devido ao seu valor nutricional e por ser bem aceita pelo consumidor. Entretanto, devido à sua alta perecibilidade, é necessário o desenvolvimento de técnicas de conservação, como a secagem, aumentando a estabilidade e distribuição desse fruto e, desenvolvendo produtos diferenciados. A modelagem matemática durante a secagem fornece informações sobre o processo em que é aplicada. O objetivo desse trabalho foi avaliar o ajuste das três equações empíricas (Henderson \& Pabis, Midilli-Kuçuk e Page) aos dados experimentais da secagem para diferentes estádios de maturação de banana (verde, madura e muito madura). Obtiveram-se bons ajustes para todas as equações, sendo a de MidilliKuçuk o melhor para representação da cinética de secagem da banana verde. A equação de Page foi a melhor para dados de banana madura e muito madura, com elevados valores de $\mathrm{R}^{2}$ e baixos valores de EQM e $\chi^{2}$.
\end{abstract}

\section{INTRODUÇÃO}

A banana (Musa ssp.) é um fruto popular em grande parte do mundo, sendo muito consumida no Brasil. A produção mundial de bananas no ano de 2014 atingiu 114 milhões de toneladas, o que torna a banana uma das frutas com maior potencial de produção global (FAO, 2017). A produção nacional representou 6\% deste total. Além disto, se destaca por possuir bom valor nutricional, sendo fonte de carboidratos e sais minerais como potássio, fósforo, magnésio, cálcio, cloro, enxofre e vitaminas $\mathrm{A}, \mathrm{B}_{1}, \mathrm{~B}_{2}, \mathrm{C}$ e $\mathrm{B}_{3}$ (niacina), todas elas de grande importância para o metabolismo humano (Padovani, 1989). Entretanto, a banana apresenta um processo acelerado de deterioração, devido seu alto teor de umidade, o que torna a comercialização do fruto in natura bastante difícil após o amadurecimento (Cabrera-Padilla et al., 2014). Diante desse contexto, a utilização da secagem se apresenta como um processo interessante do ponto de vista econômico e comercial.

A secagem é uma operação unitária que envolve as transferências de calor e massa, utilizada comumente com intuito de estender a vida útil de matérias primas e produtos alimentícios. A diminuição do teor de umidade e atividade de água do material está diretamente relacionada à queda da atividade microbiológica e de reações químicas, bioquímicas e físicas que podem ocorrer durante o armazenamento (Resende et al., 2008). No processo de secagem, obtém-se uma relação entre o teor de umidade da amostra e o tempo. 
Matematicamente é possível a modelagem desta relação, permitindo-se avaliar o comportamento da cinética de secagem de um material em determinadas condições.

Diante disso, objetivou-se, nesse trabalho, avaliar a cinética de secagem da banana em seus diferentes estádios de maturação, ajustando os modelos matemáticos propostos por Henderson e Pabis, Midilli-Kuçuk e Page aos dados obtidos experimentalmente.

\section{MATERIAIS E MÉTODOS}

As bananas (Musa acuminata var. Nanica) foram adquiridas em um mercado local (Campinas, Estado de São Paulo). Para minimizar a influência da variabilidade dos frutos, uma penca de bananas foi adquirida na fase verde. Os experimentos foram feitos sequencialmente à medida que as bananas progrediam de verdes para maduras e posteriormente, para muito maduras. As bananas foram classificadas de acordo com a cor da casca (Corrêa et al., 2012). Sólidos solúveis foram obtidos com auxílio de um refratômetro. Para determinar os sólidos solúveis, colocou-se uma amostra de banana em um mixer com água em uma proporção de 1:3. O suco obtido teve seus sólidos solúveis medidos no refratômetro e o resultado final foi calculado considerando a diluição. $\mathrm{O}$ teor de umidade, foi obtido em estufa à vácuo a $70^{\circ} \mathrm{C}(\mathrm{AOAC}, 2002)$. Os dados de caracterização das bananas são apresentados na Tabela 1.

Tabela 1 - Caracterização de bananas in natura em três estádios de maturação

\begin{tabular}{lcllllc}
\hline & \multicolumn{4}{c}{ Parâmetros de cor da polpa } & $\begin{array}{c}\text { Teor de } \\
\text { umidade } \\
\text { [kg água/ } \\
\text { kg sólido] }\end{array}$ & $\begin{array}{c}\text { SS [kg } \\
\text { sólido/ kg } \\
\text { fruto] }\end{array}$ \\
$\begin{array}{l}\text { Maturação } \\
\text { Cor da } \\
\text { casca** }\end{array}$ & $\mathrm{L}^{*}$ & $\mathrm{a}^{*}$ & $\mathrm{~b}^{*}$ & & \\
\hline Verde & 1 & $63.69 \pm 6.43$ & $2.81 \pm 1.16$ & $20.10 \pm 2.03$ & $2.38 \pm 0.03$ & $7.50 \pm 0.01$ \\
\hline Madura & 4 & $65.09 \pm 1.26$ & $3.49 \pm 1.22$ & $25.63 \pm 4.04$ & $2.50 \pm 0.00$ & $19.36 \pm 0.02$ \\
\hline $\begin{array}{l}\text { Muito } \\
\text { madura }\end{array}$ & 8 & $70.32 \pm 0.43$ & $-4.61 \pm 0.21$ & $25.37 \pm 0.08$ & $2.99 \pm 0.00$ & $25.34 \pm 0.01$ \\
\hline
\end{tabular}

** De acordo com Gous et al. (1987).

Os frutos foram lavados em água corrente, descascados e cortados transversalmente em fatias $\left(2,00 \times 10^{2} \mathrm{~m}\right.$ de diâmetro por $0,50 \times 10^{2} \mathrm{~m}$ de espessura) com um cortador circular de aço inoxidável e uma faca de aço inoxidável. Utilizou-se um paquímetro digital MIP/E-101 (Mitutoyo Sul Americana Ltda, São Paulo, Brasil) com precisão de 0,02 mm para medir o diâmetro e a espessura das fatias antes e após cada experimento de secagem (Corrêa et al., 2012).

O secador de túnel de policarbonato utilizado no experimento apresentava $1,70 \mathrm{~m}$ de comprimento com uma área de secção transversal de $0,07 \mathrm{~m}^{2}$. O ar entrava no túnel através de um soprador e era aquecido por resistências elétricas. Equalizadores foram usados para uniformizar o fluxo de ar. As amostras foram mantidas em suportes especialmente concebidos que permitiram o contato das amostras de frutos com o ar aquecido apenas na superfície superior. A massa das amostras foi medida por uma balança electrônica $(600 \mathrm{~g} / 0,001 \mathrm{~g})$. Um 
controlador lógico programável e um sistema de aquisição de dados controlaram a massa da amostra e a temperatura do ar ao longo do experimento. As condições do ar nos experimentos foram $60^{\circ} \mathrm{C}$ e $0,84 \mathrm{~m} \mathrm{~s}^{-1}$, com base em Braga et al. (2010). A umidade relativa do ar aquecido foi de $9 \pm 1 \%$ (Corrêa et al., 2012).

Com base nos dados experimentais, foram obtidos os teores adimensionais de umidade (MR), conforme a equação 1:

$$
M R=\frac{X_{t}-X_{e q}}{X_{0}-X_{e q}} \approx \frac{X_{t}}{X_{0}}
$$

Onde $\mathrm{X}_{\mathrm{t}}$ é o teor de umidade no tempo $(\mathrm{t}), \mathrm{X}_{0}$ o teor de umidade inicial e $\mathrm{X}_{\text {eq }}$ o teor de umidade de equilíbrio [ $\mathrm{kg} \mathrm{H}_{2} \mathrm{O} \mathrm{kg}{ }^{-1}$ b.s.]. Nas condições experimentais, o valor de $\mathrm{X}_{\mathrm{eq}}$ é relativamente pequeno em comparação a $\mathrm{X}_{0}$. Com base nisto, seu valor foi considerado zero neste estudo.

Para a avaliação da secagem em diferentes estádios de maturação da banana, foram utilizadas três equações empíricas. Essas equações são apresentadas na Tabela 2.

Tabela 2- Equações empíricas de cinética de secagem.

\begin{tabular}{llr}
\hline Modelo & Equação & Referência \\
\hline Henderson \& Pabis & $M R=a \cdot \exp (-k t)$ & Henderson \& Pabis (1961) \\
\hline Midilli - Kuçuk & $M R=a \cdot \exp \left(-k t^{n}\right)+b t$ & Midilli - Kuçuk (2002) \\
\hline Page & $M R=\exp \left(-k t^{n}\right)$ & Page (1949)
\end{tabular}

As análises estatísticas dos dados experimentais foram realizadas utilizando o software Statistica (Statistica 8.0, Statsoft Inc., Tulsa, OK). O erro quadrático médio (EQM) e quiquadrado reduzido $\left(\chi^{2}\right)$, Equações 5 e 6 , respectivamente, foram utilizados como critérios para apontar o melhor ajuste aos dados experimentais, bem como o coeficiente de determinação $\left(\mathrm{R}^{2}\right)$.

$$
\begin{aligned}
& E Q M=\sqrt{\left[\frac{1}{N} \sum_{i=1}^{N}\left(M R_{\text {pred }, i}-M R_{\text {exp }, i}\right)^{2}\right]} \\
& \chi^{2}=\sum_{i=1}^{N} \frac{\left(M R_{\text {pred }, i}-M R_{\text {exp }, i}\right)^{2}}{N-n^{\prime}}
\end{aligned}
$$

Onde $\mathrm{MR}_{\text {exp }}$ e $\mathrm{MR}_{\text {pred }}$ representam os teores de umidade adimensional experimentais e preditos, respectivamente; $\mathrm{N}$ é o número de observações e n é o número de constantes das equações. 


\section{RESULTADOS E DISCUSSÕES}

A partir dos dados experimentais, buscou-se a melhor representação da cinética de secagem utilizando os modelos matemáticos. Na tabela 3 são apresentados os parâmetros de cada modelo proposto e os critérios estatísticos usados para avaliar o melhor ajuste. Quanto maior o valor de $\mathrm{R}^{2}$ e menores valores de EQM e $\chi^{2}$, melhor o ajuste dos dados experimentais às equações empíricas (Junqueira et al., 2016).

Tabela 3 - Parâmetros dos modelos matemáticos e critérios de comparação.

\begin{tabular}{ccccccccc}
\hline Modelo & $\begin{array}{c}\text { Estádios } \\
\text { de } \\
\text { maturação }\end{array}$ & $\mathrm{R}^{2}$ & $\chi^{2}$ & $\mathrm{EQM}$ & $\mathrm{k}$ & $\mathrm{N}$ & $\mathrm{a}$ & $\mathrm{b}$ \\
\hline \multirow{2}{*}{$\begin{array}{c}\text { Henderson } \\
\text { e Pabis }\end{array}$} & $\mathrm{V}$ & 0.984 & 0.0569 & 0.0569 & 0.0002 & - & 1.2191 & - \\
\cline { 2 - 10 } & $\mathrm{M}$ & 0.989 & 0.0025 & 0.0456 & 0.0001 & - & 1.0547 & - \\
\hline \multirow{2}{*}{$\begin{array}{c}\text { Midilli- } \\
\text { Kuçuk }\end{array}$} & $\mathrm{V}$ & 0.994 & 0.0012 & 0.0324 & 0.0001 & - & 1.0464 & - \\
\cline { 2 - 10 } & $\mathrm{M}$ & 0.9898 & 0.0037 & 0.0468 & -1028.61 & 1182.79 & -1057.96 & 0.0000 \\
\hline \multirow{3}{*}{ Page } & $\mathrm{MM}$ & 0.975 & 0.0060 & 0.0654 & 3.3266 & 0.6883 & 0.0883 & 0.0000 \\
\cline { 2 - 9 } & $\mathrm{V}$ & 0.981 & 0.0048 & 0.0623 & 0.0000 & 1.1772 & - & - \\
\cline { 2 - 9 } & $\mathrm{M}$ & 0.992 & 0.0020 & 0.0380 & 0.0000 & 1.0952 & - & - \\
\hline
\end{tabular}

Onde: $\mathrm{a}, \mathrm{b}, \mathrm{n}$ e $\mathrm{k}$ representam as constantes empíricas e coeficientes das equações de secagem. V significa verde, $\mathrm{M}$, madura e MM, muito madura

De acordo com a Tabela 3, podemos observar que o modelo que obteve o melhor ajuste para a banana verde foi o proposto por Midilli-Kuçuk, com $\mathrm{R}^{2}, \chi^{2}$ e EQM iguais a 0,989 , 0,0037 e 0,0468 respectivamente, podendo seu comportamento ser visualizado na Figura 1. Esta equação empírica também apresentou bons ajustes aos dados experimentais durante a secagem por microondas de fatias de maçã (Zarein et al., 2015).

Figura 1 - MR em função do tempo para Banana verde

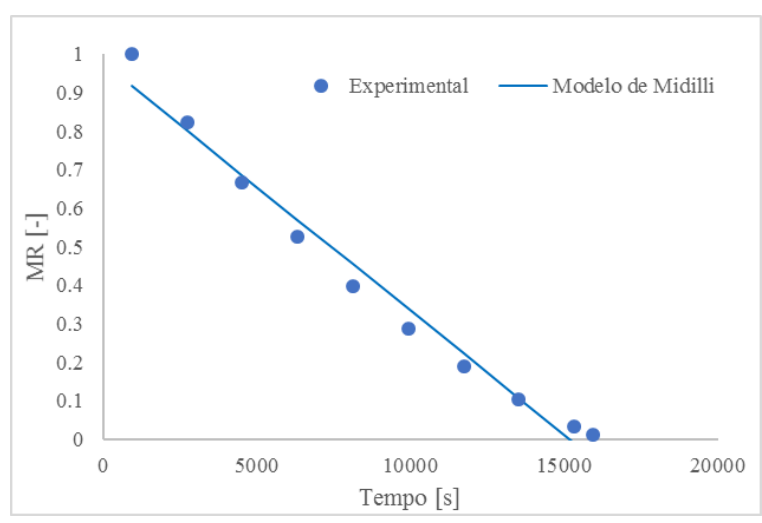

No entanto, para banana madura e banana muita madura, o modelo com melhor ajuste foi o proposto por Page, com $\mathrm{R}^{2}, \chi^{2}$ e EQM iguais a $0,992,0,0020$ e 0.0380 respectivamente para banana madura e $\mathrm{R}^{2}, \chi^{2}$ e EQM iguais a 0,995, 0,0009 e 0.0281 respectivamente para 
banana muito madura. As curvas de secagem com dados experimentais e modelados podem ser visualizadas nas Figuras 2a e 2b.

Figura 2: MR em função do tempo para Banana madura (a) e muito madura (b).
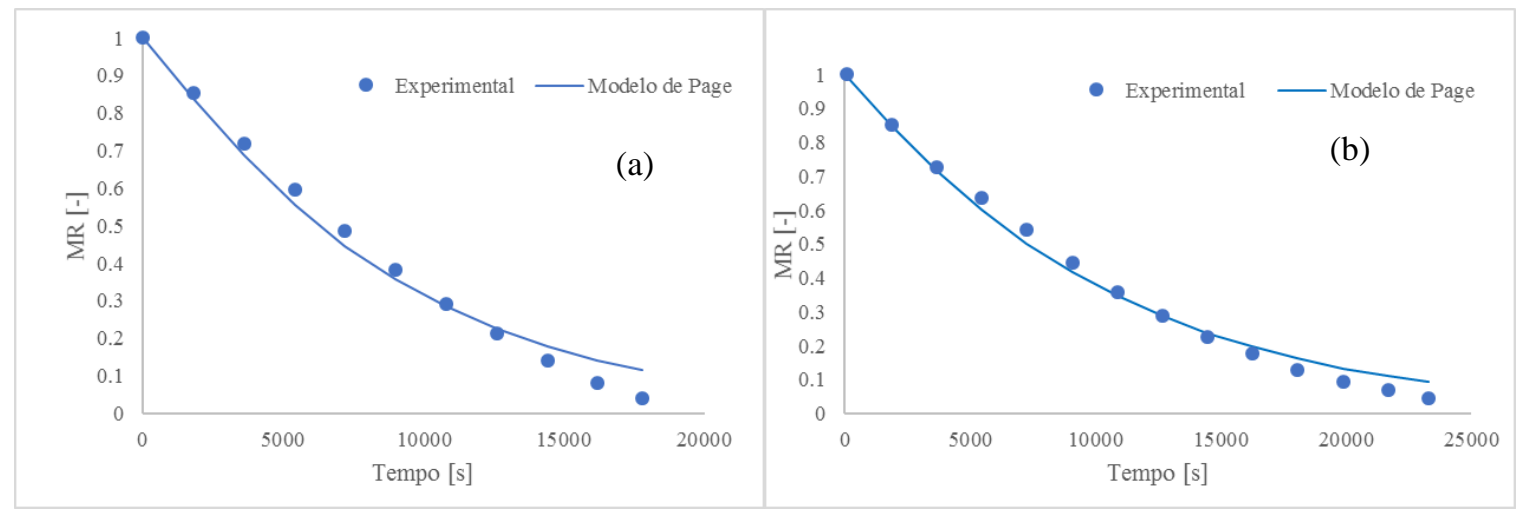

Doymaz (2012) observou que as equações que melhor se ajustaram a cinética de secagem de fatias de caqui foram as propostas por Page e Midilli-Kuçuk nas condições avaliadas.

\section{CONCLUSÃO}

Pode-se se concluir que todos os modelos tiveram bons ajustes, sendo que a banana verde o melhor foi o proposto por Midilli-Kuçuk e para banana madura e muito madura foi o proposto por Page, com altos valores de $\mathrm{R}^{2}$ e baixos valores de EQM e $\chi^{2}$.

\section{REFERÊNCIAS}

AOAC (Association of Official Analytical Chemists). Official Methods of Analysis, $18^{\text {th }}$ Ed; AOAC International: Washington, DC, 2002.

AURORE, G.; PARFAIT, B.; FAHRASMANE, L. Bananas, raw materials for making processed food products. Trends Food Sci. Tech., v. 20, p. 78-91, 2009.

CABRERA-PADILLA, R. Y.; OLADEINDE, T. O.; FINZER, J. R. D.; LIMAVERDE, J. R. Drying of banana paste in rotatory dryer with inert bed. Braz. J. Food Technol, v. 17, n. 1, p. 41-50, 2014.

CORRÊA, J. L. G.; BRAGA, A. M. P.; HOCHHEIM, M.; SILVA, A. M. The influence of ethanol on the convective drying of unripe, ripe and overripe bananas. Dry. Tech., v. 30, p. 817-826, 2012. 
DOYMAZ, I. Evaluation of some thin-layer drying models of persimmon slices. Energy Conver. Manage, v. 56, p. 199-205, 2012.

FOOD AND AGRICULTURE ORGANIZATION OF THE UNITED NATIONS - FAO. FAOSTAT Statistic Database. Disponível em: <http://www.fao.org/faostat/en/\#data/QC>. Acesso em: 29 mar. 2017.

GOUS, F.; VAN WYK, P.J.; MCGILL, E.J. The use of commercial enzymes in the processing of bananas. LWT-Food Sci. Tech., v. 20, p. 229-232, 1987.

HENDERSON, S. M.; PABIS, S. Grain drying theory I: temperature effect on drying coefficient. J. of Agri. Eng. Research, v. 6, n. 3, p. 169-174, 1961.

JUNQUEIRA, J. R. J.; MENDONÇA, K. S.; CORRÊA, J. L. J; Microwave drying of sweet potato (Ipomoea batatas (L.)) slices: Influence of the osmotic pretreatment. Def. Diff. Forum, v. 367, p. 167-174, 2016.

LII, C.Y.; CHANG, S.M.; YOUNG, Y.L. Investigation of the chemical and physical properties of banana starches. J. Food Sci., v. 47, p. 1493-1497, 1982.

MIDILLI, A.; KUCUK, H.; YAPAR, Z.A. A new model for single-layer drying. Dry. Tech., v.20, n.7, p.1503-1513, 2002

PADOVANI, M. I. Banana um Mercado Crescente Para Este Alimento Milenar. 2. ed. São Paulo: ICONE, p. 104, 1989.

PAGE, C. Factors influencing the maximum rate of drying shelled corn in layers. M.S. Thesis, Purdue University, 1949.

RESENDE, O.; FERREIRA, L. U.; ALMEIDA, D. P. Modelagem matemática para descrição da cinética de secagem do feijão adzuki (Vigna angularis). R. Bras. Prod. Agro., v. 12, n. 2, p. 171-178, 2010.

ZAREIN, M.; SAMADI, S. H.; GHOBADIAN, B. Investigation of microwavedryer effect on energy efficiency during drying of apples slices. J. Saudi Soc. Agri. Sci, v.14, p. 41-47, 2015. 